\title{
TITLE:
}

\section{Cross response in non-equilibrium systems}

$\operatorname{AUTHOR}(\mathrm{S})$ :

Ueda, M; Ohta, T

\section{CITATION:}

Ueda, M ...[et al]. Cross response in non-equilibrium systems. Journal of Statistical Mechanics: Theory and Experiment 2012, 2012(09): P09005.

ISSUE DATE:

2012-09-14

URL:

http://hdl.handle.net/2433/168392

\section{RIGHT:}

C 2012 IOP Publishing; この論文は出版社版でありません。引用の際に は出版社版をご確認ご利用ください。; This is not the published version. Please cite only the published version. 


\title{
Cross response in non-equilibrium systems
}

\author{
M. Ueda* and T. Ohta ${ }^{\dagger}$ \\ Department of Physics, Kyoto University, Kyoto 606-8502, Japan
}

(Dated: January 17, 2013)

\begin{abstract}
We study the properties of fluctuations in non-equilibrium systems focusing our attention on the cross correlations in response to external forcing. Two typical non-equilibrium systems are considered. One is a small coupled set of systems, each of which contacts a different heat bath. The other is an oscillatory system where energy input is balanced with dissipation due to nonlinear friction. Based on the Langevin equations for these systems, we investigate the non-equilibrium statistical properties such as violation of the Clausius relation in the slow change of the parameter, and fluctuations and response around the stationary state. Our main concern is the role played by the probability current.
\end{abstract}

*ueda@ton.scphys.kyoto-u.ac.jp

†takao@scphys.kyoto-u.ac.jp 


\section{INTRODUCTION}

To elucidate the properties of fluctuations out of equilibrium is one of the most fundamental problems in statistical physics. The fluctuation-dissipation theorem and the linear response theory have been formulated near thermal equilibrium based on the time-reversal symmetry and the detailed balance [1]. Substantial progress has been made recently to extend the theory to systems far from equilibrium to obtain such as fluctuation theorem [2,3], Jarzynski equality $[4,5]$ and Hatano-Sasa equality [6]. It has been shown that the path probability for both forward and backward processes is useful to understand these relations in stochastic dissipative systems [7].

In a stochastic system with non-conservative force, it has been shown that the linear response relation around the steady state is expressed in terms of the heat dissipation rate [8]. The relation has been measured experimentally $[9,10]$. In a similar stochastic system with a non-conservative force, a formula of the diffusion constant under non-equilibrium condition has been derived [11] and tested experimentally [12]. However, these theoretical results have been derived for the response to a small change of the force which maintains the system far-from-equilibrium. In this sense, the theories are limited essentially to one degree of freedom of the relevant variable.

Since violation of the detailed balance out of equilibrium is equivalent with existence of the probability current, it is of importance to explore the properties of the probability current [13]. Although fluctuation-dissipation relations and fluctuation-response relation have been studied for multi-variable systems extensively [14-22], study of the probability current has been limited. In fact, among the articles cited above, the role of the probability current has been investigated explicitly only in refs. $[14,21]$ though it was mentioned in refs. [16, 17, 20]. For example, the Harada-Sasa relation [8] has not been extended to non-variational multi-variable systems. This is probably due to the fact that one has to consider the cross responses which are not represented by a scalar variable such as the heat dissipation rate but a tensor variable.

In the present paper, we address this problem. The formulation is similar to that in ref. [14] but we take concrete systems to investigate statistical properties in non-equilibrium steady states. One is a response from a system subjected to a steady heat current. We consider a coupled system, each of which contacts a different heat bath in such a way that 
the system is subjected to a heat current. This system is represented by a set of overdamped Langevin equations with different noise intensities. The response matrix is derived around a steady state. The other is the Rayleigh oscillator which is an underdamped oscillatory system where a limit cycle oscillation is sustained by a balance of energy input and dissipation due to nonlinear friction. We shall study the response of the oscillatory stationary state to the mechanical perturbation.

Our main concern is to show explicitly how the probability current in the Fokker-Planck equation enters in violation of the linear response relation, violation of the Clausius relation and violation of the Onsager reciprocal relation by focusing our attention not only on the diagonal parts of the response matrix but also on the cross response [23]. We introduce the probability current density tensor in analogy with the stress tensor in fluid dynamics [24]. It will be shown that this tensor (not necessarily symmetric) plays an important role in the off-diagonal part of the response function around the steady state. It is mentioned here that in ref. [21], the probability current is related to the real stress tensor by considering colloidal suspensions under shear flow.

The present paper is organized as follows. In section II, we introduce a set of Langevin equations with two degrees of freedom. Equation for the probability current is derived, which contains the probability current density tensor. In section III, we identify the quantities in the steady state thermodynamics [25] and the violation of the Clausius relation is given explicitly in terms of the probability current. In section IV, we investigate some properties of fluctuations and response of the system with steady heat current. In section $\mathrm{V}$, we describe the Rayleigh oscillator with noises and study statistical properties of the system, and in section VI we present the relations of fluctuations and response in the oscillatory system. The results obtained are summarized and discussed in section VII. In Appendix A, useful formulas in the calculation of correlation functions are derived. In Appendix B, the correction to the Clausius relation is calculated explicitly for a simplified model. In Appendix $\mathrm{C}$, the relation between the violation of the Clausius relation and the previous studies is given. Any of the products of stochastic quantities are interpreted as the Stratonovich calculus throughout the present paper. 


\section{SYSTEM WITH THERMAL CURRENT}

We consider a coupled small system, each of which contacts a heat bath with different temperatures [26]. Equations of motion are given by the following Langevin equations

$$
\begin{aligned}
& \gamma_{1} \dot{x}_{1}(t)=F_{1}(\boldsymbol{x}(t) ; a(t))+\xi_{1}(t), \\
& \gamma_{2} \dot{x}_{2}(t)=F_{2}(\boldsymbol{x}(t) ; a(t))+\xi_{2}(t),
\end{aligned}
$$

where $x_{i}$ stands for the position of the $i$-th particle. The positive coefficient $\gamma_{i}$ is a friction constant and the random forces $\xi_{i}(t)$ are assumed to obey the Gaussian white statistics;

$$
\begin{aligned}
\left\langle\xi_{i}(t)\right\rangle & =0 \\
\left\langle\xi_{i}(t) \xi_{j}\left(t^{\prime}\right)\right\rangle & =2 \gamma_{i} k_{B} T_{i} \delta_{i, j} \delta\left(t-t^{\prime}\right)
\end{aligned}
$$

with $k_{B}$ the Boltzmann constant and $T_{i}$ temperature. In a variational system, the force $F_{i}$ is given by the gradient of a potential as

$$
F_{i}=-\frac{\partial V}{\partial x_{i}}
$$

If the system is variational, that is, the system has a conservative force, and if the fluctuationdissipation relation (4) holds with $T_{1}=T_{2}$, the system satisfies the detailed balance condition. The system (1) and (2) with a harmonic potential $V=(k / 2)\left(x_{1}-x_{2}\right)^{2}$ with $k$ a positive constant has been studied [27]. Here we consider more general situations. There are basically two cases. One is the system of thermal ratchets [8, 28] where eqs. (1) and (2) have a finite solution of the average velocity $\left\langle\dot{x}_{i}(t)\right\rangle$. The other is the case that this set of equations has a stable fixed point solution in the absence of the noise terms. The force may contain a nonconservative part such as $\partial F_{i} / \partial x_{j} \neq \partial F_{j} / \partial x_{i}$ for $i \neq j$. We do not consider a limit cycle oscillation which will be investigated separately in sections V and VI. The force $F_{i}$ is assumed to have a time-dependent parameter $a(t)$ so that the system executes some work by changing $a(t)$. This is realized experimentally, e.g., by optical tweezer of colloid particles [9, 29].

The Fokker-Planck equation corresponding to eqs. (1) and (2) is given by [30]

$$
\frac{\partial P}{\partial t}(\boldsymbol{x}, t)=-\sum_{i=1,2} \frac{1}{\gamma_{i}} \frac{\partial}{\partial x_{i}}\left[F_{i}(\boldsymbol{x} ; a(t)) P(\boldsymbol{x}, t)\right]
$$




$$
\begin{gathered}
+\sum_{i=1,2} \frac{1}{\gamma_{i} \beta_{i}} \frac{\partial^{2}}{\partial x_{i}^{2}} P(\boldsymbol{x}, t) \\
=-\frac{\partial}{\partial x_{1}} J_{1}(\boldsymbol{x}, t)-\frac{\partial}{\partial x_{2}} J_{2}(\boldsymbol{x}, t),
\end{gathered}
$$

where $\beta_{i}=1 / k_{B} T_{i}$ and the probability currents are defined by $[13,31]$

$$
J_{i}(\boldsymbol{x}, t) \equiv \frac{1}{\gamma_{i}} F_{i}(\boldsymbol{x} ; a(t)) P(\boldsymbol{x}, t)-\frac{1}{\gamma_{i} \beta_{i}} \frac{\partial P}{\partial x_{i}}(\boldsymbol{x}, t) .
$$

We do not consider the case of cross diffusion $\partial^{2} P /\left(\partial x_{i} \partial x_{j}\right)(i \neq j)$ in the Fokker-Planck equation. Note that the integral of $J_{i}$ is given by

$$
\left\langle\dot{x}_{i}(t)\right\rangle=\int d \boldsymbol{x} J_{i}(\boldsymbol{x}, t)=\left\langle\nu_{i}(t)\right\rangle,
$$

where

$$
\nu_{i}(\boldsymbol{x}, t) \equiv \frac{1}{\gamma_{i}} F_{i}(\boldsymbol{x} ; a(t))-\frac{1}{\gamma_{i} \beta_{i}} \frac{1}{P(\boldsymbol{x}, t)} \frac{\partial P}{\partial x_{i}}(\boldsymbol{x}, t) .
$$

Equation (8) indicates that the average of the probability current vanishes $\left\langle\nu_{i}(t)\right\rangle=0$ in a steady state when the set of eqs. (1) and (2) has a stable fixed point in the absence of the noise terms. However, it will be shown below that the probability current multiplied by other quantities such as $J_{i} F_{j}$ plays a central role in non-equilibrium states.

The probability current $J_{i}$ satisfies

$$
\begin{aligned}
\frac{\partial}{\partial t} J_{i}(\boldsymbol{x}, t)= & -\sum_{j} \frac{\partial}{\partial x_{j}} \Pi_{i j}(\boldsymbol{x}, t) \\
& +\sum_{j} J_{j}(\boldsymbol{x}, t) \frac{1}{\gamma_{i}} \frac{\partial F_{i}}{\partial x_{j}}(\boldsymbol{x} ; a(t)) \\
& +\frac{1}{\gamma_{i}} \dot{a}(t) \frac{\partial F_{i}}{\partial a}(\boldsymbol{x} ; a(t)) P(\boldsymbol{x}, t),
\end{aligned}
$$

where the probability current tensor $\Pi_{i j}$ is introduced as

$$
\begin{aligned}
\Pi_{i j}(\boldsymbol{x}, t) \equiv & \frac{1}{\gamma_{i}} F_{i}(\boldsymbol{x} ; a(t)) J_{j}(\boldsymbol{x}, t) \\
& -\frac{1}{\gamma_{i} \beta_{i}} \frac{\partial}{\partial x_{i}} J_{j}(\boldsymbol{x}, t) .
\end{aligned}
$$

This tensor is not generally symmetric. In fact, substituting eq. (7) into eq. (11), we obtain

$$
\begin{aligned}
\Pi_{i j}= & \frac{F_{i} F_{j}}{\gamma_{i} \gamma_{j}} P-\frac{F_{i}}{\gamma_{i} \gamma_{j} \beta_{j}} \frac{\partial P}{\partial x_{j}}-\frac{F_{j}}{\gamma_{i} \gamma_{j} \beta_{i}} \frac{\partial P}{\partial x_{i}} \\
& -\frac{1}{\gamma_{i} \gamma_{j} \beta_{i}} \frac{\partial F_{j}}{\partial x_{i}} P+\frac{1}{\gamma_{i} \gamma_{j} \beta_{i} \beta_{j}} \frac{\partial^{2} P}{\partial x_{i} \partial x_{j}},
\end{aligned}
$$


and

$$
\Pi_{i j}-\Pi_{j i}=-\frac{1}{\gamma_{i} \gamma_{j} \beta_{i}} \frac{\partial F_{j}}{\partial x_{i}} P+\frac{1}{\gamma_{j} \gamma_{j} \beta_{j}} \frac{\partial F_{i}}{\partial x_{j}} P
$$

If the force satisfies the relation (5) and $\beta_{i}=\beta_{j},(13)$ is identically equal to zero. We will show in section IV that the integral of (12) and (13) over $\boldsymbol{x}$ is accessible experimentally. It is also noted that the existence of the second and third terms in eq. (10) implies that the probability current is not a conserved quantity. This is because the integral of the current is equal to $\left\langle\dot{x}_{i}(t)\right\rangle$ as eq. (8) and the variables $x_{i}$ obey the dissipative dynamics. We assume that the real part of the eigenvalues of the matrix $\partial_{i} F / \partial x_{j}$ is negative provided that the stationary solution of eqs. (1) and (2) without noise terms is stable.

According to the stochastic energetics [27], the stochastic heat flowing from the $i$-th heat bath to the system per unit time can be represented as

$$
\begin{aligned}
\dot{Q}_{i}(t) & \equiv\left[-\gamma_{i} \dot{x}_{i}(t)+\xi_{i}(t)\right] \dot{x}_{i}(t) \\
& =-F_{i}(\boldsymbol{x} ; a(t)) \dot{x}_{i}(t) .
\end{aligned}
$$

Therefore, the total stochastic heat flowing from the $i$-th heat bath to the system in the time interval $[0, t]$ is given by

$$
Q_{i}=-\int_{0}^{t} d s F_{i}(\boldsymbol{x}(s), s) \dot{x}_{i}(s)
$$

The average of this with respect to the stochastic noises gives us

$$
\begin{aligned}
\left\langle Q_{i}\right\rangle & =-\int_{0}^{t} d s \int d \boldsymbol{x}\left\langle F_{i}(\boldsymbol{x}, s) \dot{x}_{i}(s) \delta(\boldsymbol{x}-\boldsymbol{x}(s))\right\rangle \\
& =-\int_{0}^{t} d s \int d \boldsymbol{x} F_{i}(\boldsymbol{x}, s) J_{i}(\boldsymbol{x}, s) .
\end{aligned}
$$

In this derivation we have used the formula (A2) in Appendix A. When the system is variational as eq. (5) and $a$ is constant, we obtain

$$
\begin{gathered}
\left\langle\dot{Q}_{1}\right\rangle+\left\langle\dot{Q}_{2}\right\rangle=\left\langle\frac{d V}{d t}\right\rangle=0, \\
\sum_{i=1,2} \int d \boldsymbol{x} \frac{\partial V}{\partial x_{i}}(\boldsymbol{x}, s) J_{i}(\boldsymbol{x}, s)=0 .
\end{gathered}
$$

These relations will be used in the subsequent sections. 
The Shannon entropy is introduced as

$$
S(t) \equiv-k_{B} \int d \boldsymbol{x} P(\boldsymbol{x}, t) \log P(\boldsymbol{x}, t)
$$

so that the time-derivative of the entropy is given by

$$
\begin{aligned}
\frac{d}{d t} S(t)= & -k_{B} \int d \boldsymbol{x} \frac{\partial P}{\partial t}(\boldsymbol{x}, t) \log P(\boldsymbol{x}, t) \\
= & k_{B} \int d \boldsymbol{x}\left[\frac{\partial}{\partial x_{1}} J_{1}(\boldsymbol{x}, t)+\frac{\partial}{\partial x_{2}} J_{2}(\boldsymbol{x}, t)\right] \\
& \times \log P(\boldsymbol{x}, t) \\
= & -\sum_{i} k_{B} \beta_{i} \int d \boldsymbol{x} F_{i}(\boldsymbol{x} ; a(t)) \\
& \times\left[\frac{1}{\gamma_{i}} F_{i}(\boldsymbol{x} ; a(t)) P(\boldsymbol{x}, t)-\frac{1}{\gamma_{i} \beta_{i}} \frac{\partial P}{\partial x_{i}}(\boldsymbol{x}, t)\right] \\
& +\sum_{i} k_{B} \gamma_{i} \beta_{i} \int d \boldsymbol{x} \frac{1}{P(\boldsymbol{x}, t)} \\
& \times\left[\frac{1}{\gamma_{i}} F_{i}(\boldsymbol{x} ; a(t)) P(\boldsymbol{x}, t)-\frac{1}{\gamma_{i} \beta_{i}} \frac{\partial P}{\partial x_{i}}(\boldsymbol{x}, t)\right]^{2} \\
= & -\sum_{i} k_{B} \beta_{i} \int d \boldsymbol{x} F_{i}(\boldsymbol{x} ; a(t)) J_{i}(\boldsymbol{x}, t) \\
& +\sum_{i} k_{B} \gamma_{i} \beta_{i} \int d \boldsymbol{x} \frac{1}{P(\boldsymbol{x}, t)} J_{i}(\boldsymbol{x}, t)^{2}
\end{aligned}
$$

By using the current $\nu_{i}$ defined by eq. (9), this can be written as

$$
\begin{aligned}
\frac{d}{d t} S(t)= & -\sum_{i} k_{B} \beta_{i}\left\langle F_{i}(\boldsymbol{x} ; a(t)) \nu_{i}(\boldsymbol{x}, t)\right\rangle \\
& +\sum_{i} k_{B} \gamma_{i} \beta_{i}\left\langle\nu_{i}(\boldsymbol{x}, t)^{2}\right\rangle .
\end{aligned}
$$

The first term on the right hand side is the entropy current from heat baths (17) whereas the second term is the entropy production rate of the whole system. The relation (21) has been derived for a multi-variable case [32] as well as a single variable case [27]. The expression of the entropy change (22) will be used in the sections below.

\section{VIOLATION OF THE CLAUSIUS RELATION}

In this section, we focus on the role of probability current in the violation of the Clausius equality in steady state thermodynamics. The main result is (45) with (48). 
Now we consider the quasi-static process by changing $a(t)$ from $a_{i}$ to $a_{f}$ during the time interval $[0, \tau]$. In order to make the quasi-static process explicit, we introduce

$$
\begin{aligned}
s & \equiv \frac{t}{\tau}, \\
P(\boldsymbol{x}, t) & \equiv \hat{P}(\boldsymbol{x}, s), \\
a(t) & \equiv \hat{a}(s),
\end{aligned}
$$

and take the limit $\tau \rightarrow \infty$ [33]. In terms of the scaled variables, the Fokker-Planck equation (6) can be written as

$$
\begin{aligned}
\frac{1}{\tau} \frac{\partial \hat{P}}{\partial s}(\boldsymbol{x}, s)= & -\sum_{i=1,2} \frac{1}{\gamma_{i}} \frac{\partial}{\partial x_{i}}\left[F_{i}(\boldsymbol{x} ; \hat{a}(s)) \hat{P}(\boldsymbol{x}, s)\right] \\
& +\sum_{i=1,2} \frac{1}{\gamma_{i} \beta_{i}} \frac{\partial^{2}}{\partial x_{i}^{2}} \hat{P}(\boldsymbol{x}, s) \\
= & -\frac{\partial}{\partial x_{1}} \hat{J}_{1}(\boldsymbol{x}, s)-\frac{\partial}{\partial x_{2}} \hat{J}_{2}(\boldsymbol{x}, s) .
\end{aligned}
$$

We expand the solution and the current in powers of $1 / \tau$ as

$$
\begin{aligned}
\hat{P}(\boldsymbol{x}, s)= & \hat{P}^{(0)}(\boldsymbol{x}, s)+\frac{1}{\tau} \hat{P}^{(1)}(\boldsymbol{x}, s) \\
& +\frac{1}{\tau^{2}} \hat{P}^{(2)}(\boldsymbol{x}, s)+\cdots, \\
\hat{J}_{i}(\boldsymbol{x}, s)= & \hat{J}_{i}^{(0)}(\boldsymbol{x}, s)+\frac{1}{\tau} \hat{J}_{i}^{(1)}(\boldsymbol{x}, s) \\
& +\frac{1}{\tau^{2}} \hat{J}_{i}^{(2)}(\boldsymbol{x}, s)+\cdots,
\end{aligned}
$$

where $\hat{J}_{i}^{(n)}$ is given explicitly by

$$
\begin{aligned}
\hat{J}_{i}^{(n)}(\boldsymbol{x}, s) \equiv & \frac{1}{\gamma_{i}} F_{i}(\boldsymbol{x} ; \hat{a}(s)) \hat{P}^{(n)}(\boldsymbol{x}, s) \\
& -\frac{1}{\gamma_{i} \beta_{i}} \frac{\partial}{\partial x_{i}} \hat{P}^{(n)}(\boldsymbol{x}, s) .
\end{aligned}
$$

It is noted that the normalization of the distributions is defined by

$$
\begin{aligned}
& \int d \boldsymbol{x} \hat{P}^{(0)}(\boldsymbol{x}, s)=1 \\
& \int d \boldsymbol{x} \hat{P}^{(n)}(\boldsymbol{x}, s)=0 \quad(n \geq 1) .
\end{aligned}
$$

The zero-th order solution is given by the stationary solution for fixed values of $a$

$$
\hat{P}^{(0)}(\boldsymbol{x}, s)=P_{s s}(\boldsymbol{x} ; \hat{a}(s)) .
$$


The first order solution should obey

$$
\begin{aligned}
\frac{\partial}{\partial s} \hat{P}^{(0)}(\boldsymbol{x}, s)= & -\sum_{i=1,2} \frac{1}{\gamma_{i}} \frac{\partial}{\partial x_{i}}\left[F_{i}(\boldsymbol{x} ; \hat{a}(s)) \hat{P}^{(1)}(\boldsymbol{x}, s)\right] \\
& +\sum_{i=1,2} \frac{1}{\gamma_{i} \beta_{i}} \frac{\partial^{2}}{\partial x_{i}^{2}} \hat{P}^{(1)}(\boldsymbol{x}, s) \\
= & -\frac{\partial}{\partial x_{1}} \hat{J}_{1}^{(1)}(\boldsymbol{x}, s)-\frac{\partial}{\partial x_{2}} \hat{J}_{2}^{(1)}(\boldsymbol{x}, s) .
\end{aligned}
$$

The entropy change in this quasi-static process can be evaluated from eq. (21) as follows

$$
\begin{aligned}
S(\tau)-S(0)= & -k_{B} \int_{0}^{1} d s \int d \boldsymbol{x}\left[\frac{\partial}{\partial s} \hat{P}^{(0)}(\boldsymbol{x}, s)\right] \\
& \times \log \hat{P}^{(0)}(\boldsymbol{x}, s)+\mathcal{O}\left(\frac{1}{\tau}\right) \\
= & -k_{B} \int_{0}^{1} d s \frac{d}{d s} \int d \boldsymbol{x} P_{s s}(\boldsymbol{x} ; \hat{a}(s)) \\
& \times \log P_{s s}(\boldsymbol{x} ; \hat{a}(s))+\mathcal{O}\left(\frac{1}{\tau}\right) \\
= & S_{s s}\left(a_{f}\right)-S_{s s}\left(a_{i}\right)+\mathcal{O}\left(\frac{1}{\tau}\right),
\end{aligned}
$$

where $S_{s s}(a)$ is defined by

$$
S_{s s}(a) \equiv-k_{B} \int d \boldsymbol{x} P_{s s}(\boldsymbol{x} ; a) \log P_{s s}(\boldsymbol{x} ; a)
$$

Therefore, in the limit $\tau \rightarrow \infty$, the entropy change $\Delta S$ is given from eqs. (33) and (34) by

$$
\begin{aligned}
\Delta S= & S_{s s}\left(a_{f}\right)-S_{s s}\left(a_{i}\right) \\
= & k_{B} \int_{0}^{1} d s \int d \boldsymbol{x} \\
& \sum_{i=1,2} \frac{\partial \Phi_{s s}}{\partial x_{i}}(\boldsymbol{x} ; \hat{a}(s)) \hat{J}_{i}^{(1)}(\boldsymbol{x}, s),
\end{aligned}
$$

where

$$
P_{s s}(\boldsymbol{x} ; a) \equiv e^{-\Phi_{s s}(\boldsymbol{x} ; a)} .
$$

The average heat current which flows in the $i$-th system from the heat bath is given from eq. (17) by

$$
\left\langle Q_{i}\right\rangle=-\tau \int_{0}^{1} d s \int d \boldsymbol{x} F_{i}(\boldsymbol{x} ; \hat{a}(s)) \hat{J}_{i}(\boldsymbol{x}, s)
$$


The house-keeping heat should be defined by replacing the probability current by the stationary one $[25,34]$;

$$
\begin{aligned}
&\left\langle Q_{i}^{h k}\right\rangle=-\int_{0}^{\tau} d t \int d \boldsymbol{x} F_{i}(\boldsymbol{x} ; a(t)) J_{s s i}(\boldsymbol{x}, a(t)) \\
&=-\tau \int_{0}^{1} d s \int d \boldsymbol{x} \\
& F_{i}(\boldsymbol{x} ; \hat{a}(s)) \hat{J}_{s s i}(\boldsymbol{x} ; \hat{a}(s))
\end{aligned}
$$

with $\hat{J}_{s s i}(\boldsymbol{x} ; \hat{a}(s))=\hat{J}^{(0)}(\boldsymbol{x}, s)$. It is noted here that this definition of the house-keeping heat is different from the one in refs. [6, 35, 36]. See also Appendix C. The excess heat current is a current subtracting the house-keeping heat

$$
\left\langle Q_{i}^{e x}\right\rangle \equiv\left\langle Q_{i}\right\rangle-\left\langle Q_{i}^{h k}\right\rangle
$$

By expanding the current given by eq. (38) in terms of $1 / \tau$, we obtain

$$
\begin{aligned}
\left\langle Q_{i}\right\rangle= & -\tau \int_{0}^{1} d s \int d \boldsymbol{x} F_{i}(\boldsymbol{x} ; \hat{a}(s)) \\
& \times\left\{\hat{J}_{i}^{(0)}(\boldsymbol{x}, s)+\frac{1}{\tau} \hat{J}_{i}^{(1)}(\boldsymbol{x}, s)+\cdots\right\} \\
= & \left\langle Q_{i}^{h k}\right\rangle-\int_{0}^{1} d s \int d \boldsymbol{x} F_{i}(\boldsymbol{x} ; \hat{a}(s)) \hat{J}_{1}^{(1)}(\boldsymbol{x}, s) \\
& +\mathcal{O}\left(\frac{1}{\tau}\right) .
\end{aligned}
$$

In this way, the excess heat in the limit $\tau \rightarrow \infty$ is obtained as

$$
\left\langle Q_{i}^{e x}\right\rangle=-\int_{0}^{1} d s \int d \boldsymbol{x} F_{i}(\boldsymbol{x} ; \hat{a}(s)) \hat{J}_{i}^{(1)}(\boldsymbol{x}, s) .
$$

Therefore, the excess entropy in the heat bath is given by

$$
\begin{aligned}
\left\langle\Theta^{e x}\right\rangle \equiv & -\frac{1}{T_{1}}\left\langle Q_{1}^{e x}\right\rangle-\frac{1}{T_{2}}\left\langle Q_{2}^{e x}\right\rangle \\
= & k_{B} \int_{0}^{1} d s \int d \boldsymbol{x} \\
& \sum_{i=1,2}\left[\beta_{i} F_{i}(\boldsymbol{x} ; \hat{a}(s)) \hat{J}_{i}^{(1)}(\boldsymbol{x}, s)\right] .
\end{aligned}
$$

Comparing this with (36), we note the relation

$$
\Delta S=-\left\langle\Theta^{e x}\right\rangle+\Xi
$$

where

$$
\begin{aligned}
\Xi \equiv k_{B} \int_{0}^{1} d s \int d \boldsymbol{x} \\
\sum_{i=1,2}\left[\gamma_{i} \beta_{i} \nu_{s s i}(\boldsymbol{x} ; \hat{a}(s)) \hat{J}_{i}^{(1)}(\boldsymbol{x}, s)\right]
\end{aligned}
$$


with

$$
\nu_{s s i}(\boldsymbol{x} ; \hat{a}(s)) \equiv \frac{J_{s s i}(\boldsymbol{x} ; \hat{a}(s))}{P_{s s}(\boldsymbol{x} ; \hat{a}(s))} .
$$

Equation (46) can be manipulated for the steady state as

$$
\begin{aligned}
\Xi= & k_{B} \int_{0}^{1} d s \int d \boldsymbol{x} \\
& \sum_{i=1,2} \gamma_{i} \beta_{i}\left[\nu_{s s i}(\boldsymbol{x} ; \hat{a}(s))\right]^{2} \hat{P}^{(1)}(\boldsymbol{x}, s) .
\end{aligned}
$$

This can be obtained as follows;

$$
\begin{aligned}
\nu_{s s i} \hat{J}_{i}^{(1)}= & \frac{\hat{J}_{i}^{(0)}}{\hat{P}^{(0)}}\left[\frac{1}{\gamma_{i}} F_{i} \hat{P}^{(1)}-\frac{1}{\gamma_{i} \beta_{i}} \frac{\partial \hat{P}^{(1)}}{\partial x_{i}}\right] \\
= & \frac{\hat{J}_{i}^{(0)}}{\gamma_{i} \hat{P}^{(0)}}\left[F_{i} \frac{\hat{P}^{(1)}}{\hat{P}^{(0)}} \hat{P}^{(0)}-\frac{1}{\beta_{i}} \frac{\partial}{\partial x_{i}}\left(\frac{\hat{P}^{(1)}}{\hat{P}^{(0)}} \hat{P}^{(0)}\right)\right] \\
= & \frac{\hat{J}_{i}^{(0)}}{\gamma_{i} \hat{P}^{(0)}} \frac{\hat{P}^{(1)}}{\hat{P}^{(0)}}\left[F_{i} \hat{P}^{(0)}-\frac{1}{\beta_{i}} \frac{\partial \hat{P}^{(0)}}{\partial x_{i}}\right] \\
& -\frac{\hat{J}_{i}^{(0)}}{\gamma_{i} \hat{P}^{(0)}} \hat{P}^{(0)} \frac{1}{\beta_{i}} \frac{\partial}{\partial x_{i}}\left(\frac{\hat{P}^{(1)}}{\hat{P}^{(0)}}\right) \\
= & \left(\frac{\hat{J}_{i}^{(0)}}{\hat{P}^{(0)}}\right)^{2} \hat{P}^{(1)}-\frac{\hat{J}_{i}^{(0)}}{\beta_{i} \gamma_{i}} \frac{\partial}{\partial x_{i}}\left(\frac{\hat{P}^{(1)}}{\hat{P}^{(0)}}\right) .
\end{aligned}
$$

Substituting this into eq. (46) yields eq. (48). The second term in eq. (49) does not contribute to (48) after integral by part because $\sum_{i} \partial J_{i}^{(0)} / \partial x_{i}=0$ for the steady state.

The relation (45) indicates that the usual Clausius relation does not hold generally in non-equilibrium steady states. It is noted that the correction (48) is the same as the entropy production given by the second term on the right hand side of (22) with the average with respect to the distribution $\hat{P}^{(1)}(\boldsymbol{x}, s)$. In Appendix B, we show an example that the correction $\Xi$ is indeed finite. To the authors' knowledge, the relation (45) with (48) has not been derived so far. The violation is represented by the steady probability current $\nu_{s s i}$, which appears in various formulas such as the Hatano-Sasa relation [6] and the violation of the fluctuationdissipation relation in non-equilibrium steady state $[11,14,21]$. It should be noted that $\Xi$ given by (48) contains the quantity $\hat{P}^{(1)}(\boldsymbol{x}, s)$ which characterizes the relaxation to the steady state.

Quite recently, the condition that the extended Clausius relation holds has been obtained [37]. Since the formulation is quite different from the present one, the relation with the result 
(45) is not clear at present. It is also mentioned here that an extension of Jarzynski's work relation and the second law of thermodynamics has been formulated recently in a heat conducting system [38]. It has been shown that the non-equilibrium contribution to the second law is related to the violation of linear response relation for heat conduction in a weak non-equilibrium regime. We also note that a new definition of excess heat has been proposed on the basis of Minimum Entropy Production Principle recently [39], in terms of which a Clausius inequality is satisfied in non-equilibrium stochastic systems. Another Clausius inequality between a renormalized work and the free energy has also been obtained in a macroscopic fluctuation theory [40].

The same procedure of the slowness expansion described above can be applied to the total entropy change in a trajectory of the stochastic dynamics, which is a sum of the system entropy and the reservoir entropy as given by eq. (C1) in Appendix C and is decomposed as $[35,36]$

$$
\Delta s_{t o t}=\Delta s_{n a}+\Delta s_{a}
$$

Each term on the right hand side is given for the present stochastic dynamics (1) and (2) by

$$
\begin{aligned}
\Delta s_{n a} \equiv & \Phi(\boldsymbol{x}(\tau), \tau)-\Phi(\boldsymbol{x}(0), 0) \\
& -\int_{0}^{\tau} d t \sum_{i} \frac{\partial \Phi_{s s}}{\partial x_{i}}(\boldsymbol{x}(t) ; a(t)) \dot{x}_{i}(t) \\
\Delta s_{a} \equiv & \int_{0}^{\tau} d t \sum_{i}\left[\beta_{i} F_{i}(\boldsymbol{x}(t) ; a(t))+\frac{\partial \Phi_{s s}}{\partial x_{i}}(\boldsymbol{x}(t) ; a(t))\right] \dot{x}_{i}(t) \\
= & \int_{0}^{\tau} d t \sum_{i} \gamma_{i} \beta_{i} \nu_{s s, i}(\boldsymbol{x}(t) ; a(t)) \dot{x}_{i}(t)=\tilde{\Theta}_{h k}
\end{aligned}
$$

where $\Phi(\boldsymbol{x}, t) \equiv-\log P(\boldsymbol{x}, t)$ and we have used eq. (C5) in Appendix C. As shown there, the average of the non-adiabatic part $\Delta s_{n a}$ is, at most, of the order of $1 / \tau$ whereas the average of the adiabatic part $\Delta s_{a}$ is given by

$$
\begin{aligned}
\left\langle\Delta s_{a}\right\rangle= & \tau \int_{0}^{1} d s \sum_{i} \gamma_{i} \beta_{i} \int d \boldsymbol{x} \nu_{s s, i}(\boldsymbol{x} ; \hat{a}(s)) J_{s s, i}(\boldsymbol{x} ; \hat{a}(s)) \\
& +\Xi+\mathcal{O}\left(\frac{1}{\tau}\right)
\end{aligned}
$$

where $\Xi$ is given by eq. (48). Therefore, $\Delta s_{t o t}$ has a term proportional to $\tau$ as eq. (53), which comes from the housekeeping part of the entropy change of heat baths as should be. 


\section{RESPONSE FUNCTION AROUND THE STEADY STATE}

In this section, we study the property of the response function matrix around the nonequilibrium steady state to derive the main formula (64) in terms of the probability current tensor.

We investigate a linear response of the system with a steady heat current by adding an external force $f_{i}(t)$ in the Langevin equation

$$
\gamma_{i} \dot{x}_{i}(t)=F_{i}(\boldsymbol{x}(t))+\xi_{i}(t)+f_{i}(t)
$$

The linear response of a quantity $A(t)$ is defined by as

$$
\left.\frac{\partial}{\partial f_{j}\left(t^{\prime}\right)}\langle A(t)\rangle_{f}\right|_{f_{j}=0}=R_{A, f_{j}}\left(t ; t^{\prime}\right) .
$$

Since both $f_{i}$ and $\xi_{i}$ are linear in eq. (54), the response function can be obtained by using the Novikov theorem (A1) as [14]

$$
R_{A, f_{j}}\left(t ; t^{\prime}\right)=\frac{1}{2 \gamma_{j} k_{B} T_{j}}\left\langle A(t)\left[\gamma_{j} \dot{x}_{j}\left(t^{\prime}\right)-F_{j}\left(\boldsymbol{x}\left(t^{\prime}\right)\right)\right]\right\rangle_{0}
$$

where $\langle\cdots\rangle_{0}$ means the average in the absence of the external force.

In particular, when we put $A(t)=\dot{x}_{i}(t)$ and $t^{\prime}=t$, eq. (56) becomes

$$
\begin{gathered}
R_{\dot{x}_{i}, f_{j}}(t ; t)=\frac{1}{2 \gamma_{j} k_{B} T_{j}}\left\langle\dot { x } _ { i } ( t ) \left[\gamma_{j} \dot{x}_{j}(t)\right.\right. \\
\left.\left.-F_{j}(\boldsymbol{x}(t))\right]\right\rangle_{0},
\end{gathered}
$$

where the equal-time response is defined by

$$
\begin{aligned}
R_{\dot{x}_{i}, f_{j}}(t ; t) \equiv \frac{1}{2}\left[R_{\dot{x}_{i}, f_{j}}(t ; t-0)\right. & \\
& \left.+R_{\dot{x}_{i}, f_{j}}(t ; t+0)\right] .
\end{aligned}
$$

In ref. [14], the properties of $R_{\dot{x}_{i}, f_{j}}(t ; t-0)$ have been analyzed. The diagonal parts are given by

$$
\begin{aligned}
R_{\dot{x}_{i}, f_{i}}(t ; t)= & \frac{1}{2 k_{B} T_{i}}\left\langle\dot{x}_{i}(t)^{2}\right\rangle_{0} \\
& -\frac{1}{2 \gamma_{i} k_{B} T_{i}}\left\langle\dot{x}_{i}(t) F_{i}(\boldsymbol{x}(t))\right\rangle_{0} \\
= & \frac{1}{2 k_{B} T_{i}}\left\langle\dot{x}_{i}(t)^{2}\right\rangle_{0}+\frac{1}{2 \gamma_{i} k_{B} T_{i}}\left\langle\dot{Q}_{i}\right\rangle_{0},
\end{aligned}
$$


where we have used eq. (16). The heat production given by the second term indicates the violation of the fluctuation-dissipation theorem. The relation (59) is the Harada-Sasa equality which was originally derived for a Langevin system subjected to a non-conservative force with a periodic potential [8]. It is noted that the sum of the diagonal parts is given by

$$
\begin{aligned}
& \sum_{i=1,2} 2 \gamma_{i} k_{B} T_{i} R_{\dot{x}_{i}, f_{i}}(t ; t) \\
= & \sum_{i=1,2} \gamma_{i}\left\langle\dot{x}_{i}(t)^{2}\right\rangle_{0}+\sum_{i=1,2}\left\langle\dot{Q}_{i}\right\rangle_{0} .
\end{aligned}
$$

As a special case, when the force is given by eq. (5), we obtain from eq. (18)

$$
\begin{gathered}
\sum_{i=1,2} 2 \gamma_{i} k_{B} T_{i} R_{\dot{x}_{i}, f_{i}}(t ; t)=\sum_{i=1,2} \gamma_{i}\left\langle\dot{x}_{i}(t)^{2}\right\rangle_{0} \\
\sum_{i=1,2} R_{\dot{x}_{i}, f_{i}}(t ; t)=\sum_{i=1,2} \frac{1}{2 k_{B} T_{i}}\left\langle\dot{x}_{i}(t)^{2}\right\rangle_{0}+\left[\frac{1}{2 \gamma_{1} k_{B} T_{1}}\right. \\
\left.-\frac{1}{2 \gamma_{2} k_{B} T_{2}}\right]\left\langle\dot{Q}_{1}\right\rangle_{0} .
\end{gathered}
$$

It is noted that the sum of the diagonal parts (62) has an extra additive term for $\gamma_{1} T_{1} \neq \gamma_{2} T_{2}$.

The off-diagonal part for $i \neq j$ is given by

$$
\begin{aligned}
R_{\dot{x}_{i}, f_{j}}(t ; t)= & \frac{1}{2 k_{B} T_{j}}\left\langle\dot{x}_{i}(t) \dot{x}_{j}(t)\right\rangle_{0} \\
& -\frac{1}{2 \gamma_{j} k_{B} T_{j}}\left\langle\dot{x}_{i}(t) F_{j}(\boldsymbol{x}(t))\right\rangle_{0} .
\end{aligned}
$$

Substituting eq. (1) into the second term and using the formula (A6), we obtain

$$
2 k_{B} T_{j} R_{\dot{x}_{i}, f_{j}}(t ; t)=\left\langle\dot{x}_{i}(t) \dot{x}_{j}(t)\right\rangle_{0}-\int d \boldsymbol{x} \Pi_{j i}(\boldsymbol{x}, t)
$$

where we have used the definition (11) as

$$
\begin{aligned}
\int d \boldsymbol{x} \Pi_{j i}(\boldsymbol{x}, t)= & \frac{1}{\gamma_{i} \gamma_{j}}\left\langle F_{i}(\boldsymbol{x}(t)) F_{j}(\boldsymbol{x}(t))\right\rangle_{0} \\
& +\frac{k_{B} T_{i}}{\gamma_{i} \gamma_{j}}\left\langle\frac{\partial F_{j}(\boldsymbol{x}(t))}{\partial x_{i}}\right\rangle_{0} .
\end{aligned}
$$

In the analogy of hydrodynamics, the first term in (65) is the "reversible part of the momentum flux density tensor" whereas the second term is the "viscous stress tensor" [24]. We emphasize that the result (64) enables us to measure the integral of the probability current 
tensor which is a fundamental quantity out of equilibrium. It is readily shown from eq. (64) with (65) that

$$
\begin{aligned}
& 2 k_{B} T_{2} R_{\dot{x}_{1}, f_{2}}(t ; t)-2 k_{B} T_{1} R_{\dot{x}_{2}, f_{1}}(t ; t) \\
= & -\frac{1}{\gamma_{2}}\left\langle\dot{x}_{1}(t) F_{2}(\boldsymbol{x}(t))\right\rangle_{0}+\frac{1}{\gamma_{1}}\left\langle\dot{x}_{2}(t) F_{1}(\boldsymbol{x}(t))\right\rangle_{0} \\
= & \frac{1}{\gamma_{1} \gamma_{2}}\left[k_{B} T_{2}\left\langle\frac{\partial F_{1}}{\partial x_{2}}(\boldsymbol{x}(t))\right\rangle_{0}\right. \\
& \left.\quad-k_{B} T_{1}\left\langle\frac{\partial F_{2}}{\partial x_{1}}(\boldsymbol{x}(t))\right\rangle_{0}\right] .
\end{aligned}
$$

When the system is variational as eq. (5), this is proportional to $\left(T_{1}-T_{2}\right)\left\langle\partial^{2} V / \partial x_{1} \partial x_{2}\right\rangle_{0}$ and hence provides us with information of the coupling strength of the two variables $x_{1}$ and $x_{2}$. On the other hand, when the system is non-variational, the right hand side of eq. (66) does not vanish generally even for $T_{1}=T_{2}$.

\section{LIMIT CYCLE OSCILLATION UNDER NOISES}

In this section, we consider a limit cycle oscillation subjected to stochastic noises. Recently the Jarzynski equality has been examined in the van der Pol and the Rayhleigh oscillators without noises [41]. The entropy production in a noisy limit cycle has also been investigated [42]. In a non-linear oscillatory system, there is a steady energy input to compensate dissipation due to friction so that an oscillation is sustained. In this sense, the system is subjected to energy current. Therefore, it is of fundamental importance to elucidate the response to the external perturbation in a non-equilibrium underdamped system undergoing a limit cycle oscillation and compare the properties of the over-damped system with steady heat current described in the preceding sections.

To be specific, we take the Rayleigh oscillator with noises governed by the following equations

$$
\begin{aligned}
\dot{x}(t) & =p(t), \\
\dot{p}(t) & =-k x(t)-g(p)+\xi(t), \\
\left\langle\xi(t) \xi\left(t^{\prime}\right)\right\rangle & =2 M \delta\left(t-t^{\prime}\right),
\end{aligned}
$$

with

$$
g(p)=\mu_{3} p^{3}+\mu_{1} p
$$


where $k>0, \mu_{1}<0$ and $\mu_{3}>0$ are constant and we have put the particle mass equal to 1 . The Fokker-Planck equation is given in terms of $\Gamma=(x, p)$ by

$$
\begin{aligned}
\frac{\partial P}{\partial t}(\Gamma, t)= & -p \frac{\partial}{\partial x} P(\Gamma, t)+k x \frac{\partial}{\partial p} P(\Gamma, t) \\
& +\frac{\partial}{\partial p}[g(p) P(\Gamma, t)]+M \frac{\partial^{2}}{\partial p^{2}} P(\Gamma, t) \\
\equiv & -\frac{\partial}{\partial x} J_{x}(\Gamma, t)-\frac{\partial}{\partial p} J_{p}(\Gamma, t),
\end{aligned}
$$

where

$$
\begin{aligned}
J_{x}(\Gamma, t) \equiv & p P(\Gamma, t), \\
J_{p}(\Gamma, t) \equiv & -k x P(\Gamma, t)-g(p) P(\Gamma, t) \\
& -M \frac{\partial}{\partial p} P(\Gamma, t) .
\end{aligned}
$$

The time-derivative of the Shannon entropy defined by

$$
S(t)=-k_{B} \int d \Gamma P(\Gamma, t) \log P(\Gamma, t)
$$

is given after some manipulations by

$$
\begin{aligned}
\frac{d}{d t} S(t)= & -\frac{k_{B}}{M} \int d \Gamma\left[g(p)^{2}-M \frac{\partial g(p)}{\partial p}\right] P(\Gamma, t) \\
& +\frac{k_{B}}{M} \int d \Gamma \frac{1}{P(\Gamma, t)} J^{(D)}(\Gamma, t)^{2},
\end{aligned}
$$

where

$$
J^{(D)}(\Gamma, t) \equiv-g(p) P(\Gamma, t)-M \frac{\partial P}{\partial p}(\Gamma, t)
$$

Here it is noted that a quantity similar to the probability current density tensor (11) is not useful in the present underdamped system. The first term in eq. (75) can be rewritten as

$$
\begin{aligned}
\langle\sigma(t)\rangle & \equiv \frac{1}{M} \int d \Gamma\left[g(p)^{2}-M \frac{\partial g(p)}{\partial p}\right] P(\Gamma, t) \\
& =-\frac{1}{M} \int d \Gamma g(p) J^{(D)}(\Gamma, t) .
\end{aligned}
$$

Therefore, eq. (75) becomes

$$
\frac{d}{d t} S(t)=\frac{k_{B}}{M}\left\langle g(p) \nu^{(D)}(\Gamma, t)\right\rangle+\frac{k_{B}}{M}\left\langle\nu^{(D)}(\Gamma, t)^{2}\right\rangle,
$$


where $\nu^{(D)}(\Gamma, t)=J^{(D)}(\Gamma, t) / P(\Gamma, t)$. The first term takes a form of the dissipative force $g$ times the current $\nu$ and hence this is a generalization of the heat dissipation. The second term is a generalization of the entropy production of the total system. In the steady state, we have $d S / d t=0$ so that

$$
-\frac{k_{B}}{M}\left\langle g(p) \nu_{s s}^{(D)}(\Gamma)\right\rangle=\frac{k_{B}}{M}\left\langle\nu_{s s}^{(D)}(\Gamma)^{2}\right\rangle \geq 0
$$

Thus the entropy production is positive in the steady non-equilibrium system. It is noted that the derivation of eq. (78) is independent of the form of the dissipative force $g(p)$. The difference of sign in the first term of (78) from that of (22) comes from the definition (70) of the dissipative force $g(p)$.

\section{RESPONSE OF THE LIMIT CYCLE OSCILLATION}

In this section, we investigate the property of the response of the nonlinear oscillator and identify the response function as eq. (90) in which the dissipation rate (77) enters and hence eq. (90) can be regarded as an extension of the Harada-Sasa equality to nonlinear oscillators.

We consider the case in the presence of an external force $f(t)$. We may define the energy of the particle as

$$
H(x, p) \equiv \frac{1}{2}\left[k x^{2}+p^{2}\right] .
$$

Its time derivative is given by

$$
\frac{d}{d t} H(\Gamma(t))=\dot{Q}(t)+\dot{W}(t),
$$

where

$$
\begin{aligned}
\dot{Q}(t) & \equiv-p(t) g(p(t))+p(t) \xi(t), \\
\dot{W}(t) & \equiv p(t) f(t) .
\end{aligned}
$$

The path probability of the particle is given by

$$
\begin{aligned}
\mathcal{P}_{\epsilon}[\Gamma(t)]= & J \delta[\dot{x}(t)-p(t)] \\
& \times e^{-\frac{1}{4 M} \int_{0}^{\tau} d t\{\dot{p}(t)+k x(t)+g(p(t))-f(t)\}^{2}},
\end{aligned}
$$


where we have used the fact that the Jacobian $J$ in the transformation of the variable from $\xi$ to $x$ is unchanged by adding the force. The response function of a quantity $A$ is given from (84) by

$$
R_{A, f}\left(t ; t^{\prime}\right)=\frac{1}{2 M}\left\langle A(t)\left[\ddot{x}\left(t^{\prime}\right)+k x\left(t^{\prime}\right)+g\left(p\left(t^{\prime}\right)\right)\right]\right\rangle_{0} .
$$

In particular, when $A(t)=p(t)$, eq. (85) for $t^{\prime}=t$ becomes

$$
R_{\dot{x}, f}(t ; t)=\frac{1}{2 M}\langle p(t) \xi(t)\rangle_{0}=\frac{1}{2},
$$

where we have used the formula (A6). The average of the heat dissipation is given from eqs. (82) and (86) by

$$
\langle\dot{Q}(t)\rangle_{0}=-\langle p(t) g(p(t))\rangle_{0}+M
$$

When the relaxation is linear as $g=\gamma p$ and $M=\gamma k_{B} T$, this reduces to the result in ref. [27]. We have, from (81) and (87), the relation for $f(t)=0$

$$
\left\langle\frac{d}{d t} H(\Gamma(t))\right\rangle_{0}+\langle p(t) g(p(t))\rangle_{0}=M .
$$

This means that $\langle\dot{Q}(t)\rangle_{0}=0$ in a time-independent stationary state. When $A(t)=x(t)$, the equal-time response function is given from eq. (85) by

$$
\begin{aligned}
2 M R_{x, f}(t ; t) & =\langle x(t) \ddot{x}(t)\rangle_{0}+k\left\langle x(t)^{2}\right\rangle_{0}+\langle x(t) g(p(t))\rangle_{0} \\
& =\langle x(t) \xi(t)\rangle_{0}=0
\end{aligned}
$$

where each term on the right hand side of the first equality depends generally on time for a limit cycle oscillation. This also provides a sum rule for the correlation functions. Finally, if we put $A(t)=g(t)$ in eq. (85), we have

$$
R_{g, f}(t ; t)=\frac{1}{2 M}\left\langle g(\Gamma(t))^{2}\right\rangle_{0}-\frac{1}{2}\langle\sigma(t)\rangle_{0}
$$

This enables us to measure the average of the dissipation rate $\sigma(t)$. In the linear underdamped case $g=\gamma p$, the average of $\sigma(t)$ is given from eqs. (77) and (87) by $\langle\sigma(t)\rangle_{0}=-(\gamma / M)\langle\dot{Q}(t)\rangle_{0}$ which is, of course, identically zero in thermal equilibrium. However, this dissipation rate does not generally vanish in the nonlinear case given by eqs. (67) - (70). Therefore, eq. (90) can be regarded as an extension of the Harada-Sasa equality 
to limit cycle oscillations. The response of $g=\mu_{1} p+\mu_{3} p^{3}$, however, may not be simply accessible experimentally.

Finally we show that the limit cycle oscillator can be reduced to an "overdamped system" in terms of the phase description. For concreteness, we take the Rayleigh oscillator given by eqs. (67) and (68) but the argument given below can be applied to any limit cycle oscillations. The limit cycle solution can be written as $\mathbf{X}(\phi(t))=(x(\phi(t)), p(\phi(t)))$ where $\mathbf{X}(\phi)=\mathbf{X}(\phi+2 \pi)$ and $d \phi / d t=\omega_{0}$ with $\omega_{0}$ the intrinsic frequency of the limit cycle. Now we consider the periodic external force $f(t)=\epsilon \sin (\Omega t)$ with $\epsilon$ a constant and assume that $\Omega$ is not much different from $\omega_{0}$ and the amplitude of the limit cycle relaxes sufficiently rapidly. In this situation, the phase defined by $\psi \equiv \phi-\Omega t$ is governed approximately after the time average over one period $2 \pi / \Omega$ by [43-45]

$$
\frac{d \psi}{d t}=\omega_{0}-\Omega+G(\psi)+\eta(t)
$$

where $G(\psi) \sim O(\epsilon)$ is a $2 \pi$-periodic function and $\eta(t)$ obeys Gaussian white statistics with $\langle\eta\rangle=0$. Equation (91) represents entrainment and phase slip of a limit cycle oscillation by a periodic force and noises [46]. If we regard $\omega_{0}-\Omega$ as a non-conservative force, this is a special case of the overdamped dynamics (1) and (2) and is the same form as the one studied in ref. [8] although the fluctuation-dissipation relation of second kind (4) does not hold in eq. (91).

\section{DISCUSSION}

We have investigated the response to the external force in two systems, both of which are modeled by Langevin equations. One is the overdamped two-variable system with a steady heat flow. In this case, we have introduced the probability current density tensor $\Pi_{i j}(12)$ which vanishes when the system is variational with the condition (4) and there is no heat flow, and therefore plays a fundamental role in the non-equilibrium system. In fact, we have shown that the equal-time cross response around the steady state is represented in terms of the probability current density tensor as eqs. (63) and (66). The diagonal response function is given by an extension of the Harada-Sasa equality [8]. In the present theory, we have considered an external force which is additive in the set of Langevin equations. However, it is readily generalized to other systems in the presence of heat currents, where an external 
perturbation $h_{i}(t)$ enters multiplicatively to the variational potential as $h_{i}(t)\left(\partial V / \partial x_{i}\right)[16]$.

In the overdamped case, we have derived the formula for violation of the Clausius relation (45) in a process of slowly changing parameter of the system, which is found to be related with the entropy production rate. The correction has been evaluated explicitly for a simple model system in Appendix B. The method has also been applied to the total entropy change for slow change of the system parameter as described in detail in Appendix C.

The other is a stochastic limit cycle oscillation represented by the under-damped Langevin equation. We have shown that the time-derivative of the Shannon entropy consists of two parts as eq. (78), each of which has a meaning of the generalized energy dissipation and entropy production. The response of the dissipative force $g(p)$ is related to the average of the generalized dissipation rate $\langle\sigma(t)\rangle$. If we take a steady state average of eq. (88), we obtain

$$
\langle p g(p)\rangle_{s s}=M
$$

It is worth mentioning that this is a generalization of the law of equipartition of energy in thermal equilibrium. In fact, if we consider a purely dissipative case as $g=\gamma p$ with $M=\gamma k_{B} T$, the relation (92) reduces $\left\langle p^{2}\right\rangle_{e q}=k_{B} T$.

We have studied the overdamped dynamics and the nonlinear underdamped dynamics separately. It is remarked that these two are not totally unrelated. As shown at the end of section VI, the phase description of a nonlinear oscillator gives us an overdamped equation with a non-conserved force. Therefore a unified formulation of both systems is expected to be possible. We shall return to this problem elsewhere in the future.

\section{Acknowledgements}

This work was supported by the JSPS Core-to-Core Program "International research network for non-equilibrium dynamics of soft matter" and the Grant-in-Aid for the Global COE Program "The Next Generation of Physics, Spun from Universality and Emergence" from the Ministry of Education, Culture, Sports, Science and Technology (MEXT) of Japan. TO is supported by a Grant-in-Aid for Scientific Research (C) from Japan Society for Promotion of Science. 


\section{Appendix A}

In this Appendix, we derive the formulas for the correlation functions. It is convenient and most systematic to employ the Furutsu-Novikov-Donsker theorem [47-49] for the Gaussian stochastic variables $\xi_{n}$

$$
\left\langle\xi_{k}(t) \phi(s)\right\rangle=\int_{0}^{t} d t^{\prime} \sum_{\ell}\left\langle\xi_{k}(s) \xi_{\ell}\left(t^{\prime}\right)\right\rangle\left\langle\frac{\delta \phi(t)}{\delta \xi_{\ell}\left(t^{\prime}\right)}\right\rangle,
$$

where $\phi\{\xi\}$ is a functional of $\xi_{n}$. We apply this formula for $\phi\{\xi\}=A(\mathbf{x}(t)) \delta(\mathbf{x}-\mathbf{x}(t))$ such that

$$
\begin{aligned}
& \left\langle\xi_{k}(t) A(\mathbf{x}(t)) \delta(\mathbf{x}-\mathbf{x}(t))\right\rangle \\
= & A(\mathbf{x}) \int_{0}^{t} d t^{\prime} \sum_{\ell}\left\langle\xi_{k}(t) \xi_{\ell}\left(t^{\prime}\right)\right\rangle\left\langle\frac{\delta \delta(\mathbf{x}-\mathbf{x}(t))}{\delta \xi_{\ell}\left(t^{\prime}\right)}\right\rangle \\
= & -A(\mathbf{x}) \sum_{n, \ell} M_{k \ell} \frac{\partial}{\partial x_{n}}\left\langle\delta(\mathbf{x}-\mathbf{x}(t)) \frac{\delta x_{n}(t)}{\delta \xi_{\ell}(t)}\right\rangle \\
= & -A(\mathbf{x}) \sum_{\ell} \frac{M_{k \ell}}{\gamma_{\ell}} \frac{\partial}{\partial x_{\ell}} P(\mathbf{x}, t) .
\end{aligned}
$$

In this derivation, we have put

$$
\left\langle\xi_{k}(t) \xi_{\ell}\left(t^{\prime}\right)\right\rangle=2 M_{k \ell} \delta\left(t-t^{\prime}\right)
$$

and have used the relations

$$
\int_{-\infty}^{t} \delta(t-s) d s=\frac{1}{2}
$$

and

$$
\begin{aligned}
\frac{\delta x_{n}(t)}{\delta \xi_{\ell}(t)} & =\lim _{s \rightarrow t} \frac{\delta x_{n}(t)}{\delta \xi_{\ell}(s)} \\
& =\lim _{s \rightarrow t} \frac{\delta}{\delta \xi_{\ell}(s)}\left[\frac{1}{\gamma_{n}} \int_{0}^{t} d s^{\prime} \xi_{n}\left(s^{\prime}\right)+\text { higher order }\right] \\
& =\frac{\delta_{n \ell}}{\gamma_{n}} \int_{0}^{t} d s^{\prime} \delta\left(s-s^{\prime}\right) \\
& =\frac{\delta_{n \ell}}{\gamma_{n}}
\end{aligned}
$$

where the form of eqs. (1) and (2) has been used. If we put $M_{i j}=\left(\gamma_{i} k_{B} T_{i}\right) \delta_{i j}$, we obtain from eq. (A2)

$$
\begin{aligned}
\left\langle\xi_{k}(t) A(\mathbf{x}(t))\right\rangle & =\int d \mathbf{x}\left\langle\xi_{k}(t) A(\mathbf{x}(t)) \delta(\mathbf{x}-\mathbf{x}(t))\right\rangle \\
& =k_{B} T_{k}\left\langle\frac{\partial A(\mathbf{x})}{\partial x_{k}}\right\rangle
\end{aligned}
$$




\section{Appendix B}

In this Appendix, we evaluate $\Xi$ in eq. (46). For simplicity, we put $\gamma_{1}=\gamma_{2}=\gamma$ and $\beta_{1}=\beta_{2}=\beta$ and consider the following set of equations [13, 50];

$$
\begin{aligned}
\gamma \dot{x}_{1}(t) & =-k(t) x_{1}(t)+\alpha x_{2}(t)+\xi_{1}(t), \\
\gamma \dot{x}_{2}(t) & =-k(t) x_{2}(t)-\alpha x_{1}(t)+\xi_{2}(t) .
\end{aligned}
$$

When $\alpha \neq 0$, this set of equations is not variational and there is an irreversible circulation of fluctuations [13]. We put

$$
k(t)=k_{0}+\frac{t}{\tau} k^{\prime}
$$

This is given in terms of the scaled time $s \equiv t / \tau$ by

$$
\hat{k}(s)=k_{0}+s k^{\prime}
$$

The 0-th order solution of the distribution function is given by

$$
\begin{aligned}
& \hat{P}^{(0)}(\boldsymbol{x}, s)= P_{s s}(\boldsymbol{x} ; \hat{k}(s)) \\
&=\exp \left[-\frac{1}{2} \beta \hat{k}(s)\left(x_{1}^{2}+x_{2}^{2}\right)\right. \\
&\left.\quad+\log \left(\frac{\beta \hat{k}(s)}{2 \pi}\right)\right] .
\end{aligned}
$$

The steady probability currents are simply given by

$$
\begin{aligned}
\nu_{s s 1}(\boldsymbol{x} ; \hat{k}(s)) & =\frac{\alpha}{\gamma} x_{2}, \\
\nu_{s s 2}(\boldsymbol{x} ; \hat{k}(s)) & =-\frac{\alpha}{\gamma} x_{1} .
\end{aligned}
$$

It is readily shown from eq. (33) that the first order solution of the distribution function is given by

$$
\begin{aligned}
\hat{P}^{(1)}(\boldsymbol{x}, s)= & {\left[\frac{k^{\prime} \gamma \beta}{4 \hat{k}(s)} x_{1}^{2}+\frac{k^{\prime} \gamma \beta}{4 \hat{k}(s)} x_{2}^{2}-\frac{k^{\prime} \gamma}{2 \hat{k}(s)^{2}}\right] } \\
& \times P_{s s}(\boldsymbol{x} ; \hat{k}(s)) .
\end{aligned}
$$

The distributions $\hat{P}^{(0)}$ and $\hat{P}^{(1)}$ satisfy the normalization conditions (31). Now we write $\Xi$ as $\Xi=k_{B} \int_{0}^{1} d s I(s)$ where

$$
I(s) \equiv \beta \gamma \sum_{i=1,2} \int d \boldsymbol{x}\left[\nu_{s s i}(\boldsymbol{x} ; \hat{k}(s))\right]^{2} \hat{P}^{(1)}(\boldsymbol{x}, s) .
$$


Substituting the currents (B7) and (B8), and the first order distribution (B9) into (B10), we obtain

$$
\begin{aligned}
I(s)= & \gamma \beta \int d \boldsymbol{x} \frac{\alpha^{2}}{\gamma^{2}}\left[x_{1}^{2}+x_{2}^{2}\right] \hat{P}^{(1)}(\boldsymbol{x}, s) \\
= & \frac{\beta \alpha^{2}}{\gamma} \int d \boldsymbol{x}\left(x_{1}^{2}+x_{2}^{2}\right) \\
& \times\left[\frac{k^{\prime} \gamma \beta}{4 \hat{k}(s)} x_{1}^{2}+\frac{k^{\prime} \gamma \beta}{4 \hat{k}(s)} x_{2}^{2}-\frac{k^{\prime} \gamma}{2 \hat{k}(s)^{2}}\right] P_{s s}(\boldsymbol{x} ; \hat{k}(s)) \\
= & \frac{k^{\prime} \beta^{2} \alpha^{2}}{4 \hat{k}(s)}\left[\left\langle x_{1}^{4}\right\rangle+\left\langle x_{2}^{4}\right\rangle+2\left\langle x_{1}^{2} x_{2}^{2}\right\rangle\right] \\
& -\frac{k^{\prime} \beta \alpha^{2}}{2 \hat{k}(s)^{2}}\left[\left\langle x_{1}^{2}\right\rangle+\left\langle x_{2}^{2}\right\rangle\right] \\
= & \frac{k^{\prime} \alpha^{2}}{\hat{k}(s)^{3}} .
\end{aligned}
$$

In this way, the finiteness of $\Xi$ is confirmed. Note that the correction $\Xi$ is of the order of $\alpha^{2}$.

\section{Appendix C}

In this appendix, we apply the slowness expansion to the entropy change. The total entropy change in a single trajectory of the stochastic dynamics (1) and (2) is given by

$$
\Delta s_{t o t}=\Delta \Phi+\Theta
$$

where $\Delta \Phi$ and $\Theta$ are the entropy change of the system and the heat baths, respectively and are given by

$$
\begin{aligned}
\Delta \Phi & \equiv \Phi(\boldsymbol{x}(\tau), \tau)-\Phi(\boldsymbol{x}(0), 0) \\
\Theta & \equiv \int_{0}^{\tau} d t \sum_{i} \beta_{i} F_{i}(\boldsymbol{x}(t) ; a(t)) \dot{x}_{i}(t)
\end{aligned}
$$

In ref.[6], $\Theta$ is decomposed into the housekeeping part and the excess part as

$$
\Theta=\tilde{\Theta}_{h k}+\tilde{\Theta}_{e x}
$$

where

$$
\begin{aligned}
\tilde{\Theta}_{h k} & \equiv \int_{0}^{\tau} d t \sum_{i} \gamma_{i} \beta_{i} \nu_{s s, i}(\boldsymbol{x}(t) ; a(t)) \dot{x}_{i}(t), \\
\tilde{\Theta}_{e x} & \equiv-\int_{0}^{\tau} d t \sum_{i} \frac{\partial \Phi_{s s}}{\partial x_{i}}(\boldsymbol{x}(t) ; a(t)) \dot{x}_{i}(t) .
\end{aligned}
$$


We have from eqs. (C1) and (C4), [36]

$$
\Delta s_{t o t}=\Delta s_{a}+\Delta s_{n a}
$$

where

$$
\begin{aligned}
\Delta s_{a} & =\tilde{\Theta}_{h k} \\
\Delta s_{n a} & =\Delta \Phi+\tilde{\Theta}_{e x} .
\end{aligned}
$$

The ensemble average of these entropy changes is given by

$$
\begin{aligned}
\left\langle\tilde{\Theta}_{h k}\right\rangle= & \int_{0}^{\tau} d t \sum_{i} \gamma_{i} \beta_{i} \int d \boldsymbol{x} \\
& \times \nu_{s s, i}(\boldsymbol{x} ; a(t)) J_{i}(\boldsymbol{x}, t) \\
\left\langle\tilde{\Theta}_{e x}\right\rangle= & -\int_{0}^{\tau} d t \sum_{i} \int d \boldsymbol{x} \\
& \times \frac{\partial \Phi_{s s}}{\partial x_{i}}(\boldsymbol{x} ; a(t)) J_{i}(\boldsymbol{x}, t) .
\end{aligned}
$$

Note that $(\mathrm{C} 10)$ is different from $-\sum_{i} \beta_{i}\left\langle Q_{i}^{h k}\right\rangle$ in eq. (39). See also eqs. (C14) and (C15) below. By using the same method as (41), these are expanded as

$$
\begin{aligned}
\left\langle\tilde{\Theta}_{h k}\right\rangle= & \tau \int_{0}^{1} d s \sum_{i} \gamma_{i} \beta_{i} \int d \boldsymbol{x} \nu_{s s, i}(\boldsymbol{x} ; \hat{a}(s)) J_{s s, i}(\boldsymbol{x} ; \hat{a}(s)) \\
& +\int_{0}^{1} d s \sum_{i} \gamma_{i} \beta_{i} \int d \boldsymbol{x} \nu_{s s, i}(\boldsymbol{x} ; \hat{a}(s)) \hat{J}_{i}^{(1)}(\boldsymbol{x}, s) \\
& +\mathcal{O}\left(\frac{1}{\tau}\right) \\
= & \tau \int_{0}^{1} d s \sum_{i} \gamma_{i} \beta_{i} \int d \boldsymbol{x} \nu_{s s, i}(\boldsymbol{x} ; \hat{a}(s)) J_{s s, i}(\boldsymbol{x} ; \hat{a}(s)) \\
& +\Xi+\mathcal{O}\left(\frac{1}{\tau}\right), \\
\left\langle\tilde{\Theta}_{e x}\right\rangle= & -\int_{0}^{1} d s \sum_{i} \int d \boldsymbol{x} \frac{\partial \Phi_{s s}}{\partial x_{i}}(\boldsymbol{x} ; \hat{a}(s)) \hat{J}_{i}^{(1)}(\boldsymbol{x}, s) \\
& +\mathcal{O}\left(\frac{1}{\tau}\right) \\
= & -\left[S_{s s}\left(a_{f}\right)-S_{s s}\left(a_{i}\right)\right]+\mathcal{O}\left(\frac{1}{\tau}\right) .
\end{aligned}
$$

Putting (34) and (C13) together, we obtain $\left\langle\Delta s_{n a}\right\rangle=\mathcal{O}(1 / \tau)$. Note that the extended Clausius relation $\Delta S=-\left\langle\tilde{\Theta}_{e x}\right\rangle$ is always satisfied under the definition of (C5). However, the excess entropy change of the heat reservoirs $\left\langle\tilde{\Theta}_{e x}\right\rangle$ is not a directly measurable quantity since it requires the full probability distribution. 
In section III, we have employed the decomposition of the entropy change $\langle\Theta\rangle$ as $\langle\Theta\rangle=$ $\left\langle\Theta_{h k}\right\rangle+\left\langle\Theta_{e x}\right\rangle[34]$, where

$$
\begin{aligned}
\left\langle\Theta_{h k}\right\rangle= & \int_{0}^{\tau} d t \sum_{i} \beta_{i} \int d \boldsymbol{x} F_{i}(\boldsymbol{x} ; a(t)) \\
& \times J_{s s, i}(\boldsymbol{x}, a(t)), \\
\left\langle\Theta_{e x}\right\rangle= & \int_{0}^{\tau} d t \sum_{i} \beta_{i} \int d \boldsymbol{x} F_{i}(\boldsymbol{x} ; a(t)) \\
& \times\left[J_{i}(\boldsymbol{x}, t)-J_{s s, i}(\boldsymbol{x}, a(t))\right] .
\end{aligned}
$$

Using this definition, $\left\langle\Theta_{e x}\right\rangle$ is an excess heat dissipation and is measurable. The violation of the Clausius relation in quasi-static protocol is derived as (45).

[1] Kubo R, Toda M and Hashitsume N, 1991 Statistical Physics II: Nonequilibrium Statistical Mechanics (Berlin: Springer-Verlag)

[2] Evans D J, Cohen E G D and Morriss G P, 1993 Phys. Rev. Lett. 712401

[3] Gallavotti G and Cohen E G D, 1995 Phys. Rev. Lett. 742694

[4] Jarzynski C, 1997 Phys. Rev. Lett. 782690

[5] Jarzynski C, 1997 Phys. Rev. E 565018

[6] Hatano T and Sasa S, 2001 Phys. Rev. Lett. 863463

[7] Crooks G E, 2000 Phys. Rev. E 612361

[8] Harada T and Sasa S, 2005 Phys. Rev. Lett. 95130602

[9] Toyabe S, Jiang H R, Nakamura T, Murayama Y and Sano M, 2007 Phys. Rev. E 75011122

[10] Gomez-Solano J R, Petrosyan A, Giliberto S, Chetrite R and Gawedzski K, 2009 Phys. Rev. Lett. 103040601

[11] Speck T and Seifert U, 2006 Europhys. Lett. 74391

[12] Blickle V, Speck T, Lutz C, Seifert U and Bechinger C, 2007 Phys. Rev. Lett. 98210601

[13] Tomita K and Tomita H, 1974 Prog. Theor. Phys. 511731

[14] Ohta T and Ohkuma T, 2008 J. Phys. Soc. Jpn. 77074004

[15] Prost J, Joanny J F and Parrondo J M R, 2009 Phys. Rev. Lett. 103090601

[16] Baiesi M, Maes C and Wynants B, 2009 Phys. Rev. Lett. 103010602

[17] Baiesi M, Maes C and Wynants B, 2009 J. Stat. Phys. 1371094

[18] Evans D J, Searles D J and Williams S R, 2008 J. Chem. Phys. 128014504 
[19] Martens K, Bertin E and Dorz M, 2009 Phys. Rev. Lett. 103260602

[20] Verley G, Mallick K and Lacoste D, 2011 Europhys. Lett. 9310002

[21] Seifert U and Speck T, 2010 Europhys. Lett. 8910007

[22] Shimizu A and Yuge T, 2010 J. Phys. Soc. Jpn. 79013002

[23] Andrieux D and Gaspard P, 2006 J. Chem. Phys. 121 6167, ibid, 2006 J. Chem. Phys. 125 219902

[24] Landau L D and Lifshitz E M, 1987 Fluid Mechanics (London: Elsevier)

[25] Oono Y and Paniconi M, 1998 Prog. Theor. Phys. Suppl. 13029

[26] Dhar A, 2008 Adv. Physics 57457

[27] Sekimoto K, 2010 Stochastic Energetics (Berlin: Springer)

[28] Sekimoto K, 1997 J. Phys. Soc. Jpn. 661234

[29] Kim K and Qian H, 2007 Phys. Rev. E 75022102

[30] Gardiner C, 2009 Stochastic Methods (Berlin: Springer-Verlag)

[31] Chernyak V Y, Chertkov M and Jarzynski C, 2006 J. Stat. Mech. 08 P08001

[32] Qian H, 2001 Phys. Rev. E 65016102

[33] Sekimoto K and Sasa S, 1997 J. Phys. Soc. Jpn. 663326

[34] Komatsu T S, Nakagawa N, Sasa S and Tasaki H, 2008 Phys. Rev. Lett. 100230602

[35] Speck T and Seifert U, 2005 J. Phys. A: Math. Gen. 38 L581

[36] Esposito M and Van den Broeck C, 2010 Phys. Rev. Lett. 104090601

[37] Sagawa T and Hayakawa H, 2011 Phys. Rev. E 84051110

[38] Nakagawa N, 2012 Phys. Rev. E 85051115

[39] Maes C and Netočný K, 2012 arXiv:1206.3423

[40] Bertini L, Gabrielli D, Jona-Lasinio G and Landim C, 2012 arXiv:1208.1872

[41] Hasegawa H, 2011 Phys. Rev. E 84061112

[42] Xiao T J, Hou Z and Xin H, 2008 J. Chem. Phys. 129114506

[43] Nakao H, Arai K and Kawamura Y, 2007 Phys. Rev. Lett. 98184101

[44] Teramae J N, Nakao H and Ermentrout G B, 2009 Phys. Rev. Lett. 102194102

[45] Ly C and Ermentrout G B, 2011 Physica D 240719

[46] Pikovsky A, Rosenblum M and Kurths J, 2001 Synchronization: A Universal Concept in Nonlinear Sciences (Cambridge: CUP)

[47] Furutsu K, 1963 J. Res. Natl. Bur. Stand. 67303 
[48] Novikov E A, 1963 Zh. Eksp. Theor. Fiz. 471919

[49] Donsker M, 1964 Proceedings Conference on The Theory and Applications of Analysis in Function Space (Cambridge, MA: MIT Press)

[50] Nemoto T and Sasa S, 2011 Phys. Rev. E 84061113 